\title{
Upgrade of the CMS Electromagnetic Calorimeter for High-Luminosity LHC Operation
}

\section{Francesca CAVALLARI'INFN Sezione di Roma, Italy}

E-mail: francesca.cavallarieromal.infn.it

The Electromagnetic Calorimeter (ECAL) of the Compact Muon Solenoid (CMS) experiment at the LHC is a hermetic, fine grained, homogeneous calorimeter, comprising 75848 lead tungstate scintillating crystals, located inside the CMS superconducting solenoidal magnet. It was designed to operate for a minimum of ten years at the LHC, with an instantaneous luminosity of $1 \cdot 10^{34} / \mathrm{cm}^{2} / \mathrm{s}$ and for an integrated luminosity of 500/fb. The high luminosity LHC (HL-LHC) is expected to provide an instantaneous luminosity of around $5 \cdot 10^{34} / \mathrm{cm}^{2} / \mathrm{s}$ and integrate a total luminosity of around 3000/fb by about 2035 (ten years of data taking). The evolution of the CMS ECAL at HL-LHC is being assessed. We present results of an intensive campaign of component irradiations and ageing studies, complemented by simulations of the physics performance of the aged detector. Several upgrade options are shown, including replacements of the electronics to provide greater triggering flexibility, and new endcap detectors. The latter is the subject of much ongoing $R \& D$ and several potential technologies are presented here.

The European Physical Society Conference on High Energy Physics

18-24 July, 2013

Stockholm, Sweden

* Speaker. 


\section{Introduction}

The CMS electromagnetic calorimeter (ECAL) is a homogeneous calorimeter made of 75848 lead tungstate scintillating crystals, located inside the CMS superconducting solenoidal magnet [1]. It is made of a barrel part covering the region of pseudorapidity $|\eta|<1.48$ and two endcaps, which extend the coverage up to $|\eta|<3.0$. The photo-detectors are Avalanche Photo-Diodes (APD) in the barrel and Vacuum Phototriodes (VPT) in the endcaps. Electrons and photons are typically reconstructed until $|\eta|<2.5$, the region covered by the silicon tracker, while jets are reconstructed up to $|\eta|<3$.0. The excellent energy resolution of the ECAL was optimised using the two-photon decay of the Higgs boson as a bench-mark, and it led to its discovery in this channel [2]. The ECAL energy resolution achieved during 2010-2011 is described in [B] and ranges from 1.1 to $2.6 \%$ in the barrel and 2.2 to $5 \%$ in the endcaps for photons from the Higgs boson decay.

The design of the ECAL was made to operate for ten years at the LHC, with an instantaneous luminosity of $1 \cdot 10^{34} / \mathrm{cm}^{2} / \mathrm{s}$ and for an integrated luminosity of $500 \mathrm{fb}^{-1}$. A new program is being envisaged to exploit the full potential of the LHC machine through a luminosity upgrade, in order to precisely measure the couplings and rare decays of the Higgs boson, self-interaction through the double Higgs boson production, Vector Boson Scattering, and the properties of other newly discovered particles. The high luminosity LHC (HL-LHC) is expected to provide an instantaneous luminosity of around $5 \cdot 10^{34} / \mathrm{cm}^{2} / \mathrm{s}$ and integrate a total luminosity of around 3000/fb by about 2035. The average number of concurrent interactions per bunch-crossing (pile-up) in CMS will be about 130, and the radiation levels in the detector will be six times higher than for the nominal LHC design. This is a challenge particularly for the endcap region, where the radiation levels have a strong gradient along $\eta$ and typically change by a factor 100 between $|\eta|=1.48$ and $|\eta|=3.0$. The high energy hadron fluence at $\eta=2.6$ will be $2 \cdot 10^{14} / \mathrm{cm}^{2}$ and the $\gamma$ dose will be $300 \mathrm{kGy}$ for $3000 \mathrm{fb}^{-1}$ with an expected dose rate of $30 \mathrm{~Gy} / \mathrm{h}$.

The forward region is more important at the HL-LHC, because most of the bench-mark channels involve forward jets (Vector Boson Fusion Higgs boson production, and Vector Boson Scattering). In addition, the discovery of a low mass Higgs boson constrains the particles produced in its decays to relatively low transverse energies. The detectors, and particularly the trigger system, must therefore maintain the ability to detect low transverse energy objects.

\section{ECAL longevity}

Radiation from the LHC affects crystal transparency in two ways. The $\gamma$ irradiation reduces crystals transparency, by creating shallow defects, which spontaneously recover at room temperature. The hadron damage creates clusters of lattice defects, which cause light transmission loss, impacting more severely the $\mathrm{PbWO}_{4}$ scintillation emission spectrum. The hadron damage is cumulative and permanent at room temperature. A full recovery takes place at very high temperature $\left(350^{\circ} \mathrm{C}\right)$. Following extensive studies with beam tests on highly irradiated crystal matrices, a model of the radiation effect on the crystal calorimeter performance has been developed.

The energy resolution of the ECAL can be parametrised by the following equation:

$$
\frac{\sigma(E)}{E}=\frac{a}{\sqrt{E}} \oplus \frac{b}{E} \oplus c
$$


where the first term is referred to as the stochastic term, and the second represents the electronics noise. The third is a constant term, which includes contributions from environment instabilities, intercalibration constants and light collection uniformity. Poor light collection uniformity causes variations in light output since the penetration depth fluctuates from one electromagnetic shower to another. The crystal transparency deterioration causes a reduction of scintillation light output with respect to undamaged crystals $\left(S / S_{0}\right)$ and a deterioration of the light collection uniformity along the crystal length. Consequently the energy resolution is affected in all three terms:

- a degradation of the stochastic term due to the reduced light output, proportional to $\sqrt{S_{0} / S}$

- a worsening of the noise term due to the amplification of the energy equivalent noise, proportional to $S_{0} / S$,

- a worsening of the constant term due to the increased light collection non-uniformity along the crystal.

Figure 11 shows the predicted response reduction for an electron shower of $50 \mathrm{GeV}$ as a function of $\eta$ in the endcaps for various integrated luminosities. The $\gamma$ and hadron fluence in the detector were calculated with the MARS program [П], the crystal light output was calculated with GEANT4 [5] and SLitrani [6] for the ray-tracing through the crystals. The crystal light transmission as a function of the fluence is based on [7]. The reduction in VPT signal is based on laboratory tests [8]. The model represented in Figure 1 was validated with results from test-beam measurements of heavily irradiated crystal matrices. Figure 2 shows the expected energy resolution as a function of the transverse energy for unconverted photons impacting the calorimeter at pseudorapidity $|\eta|=2.2$, for detector ageing corresponding to 500/fb and 3000/fb of integrated luminosity.

In the endcaps, maintaining the response at a level higher than $10 \%$ compared to the initial detector, will only be possible up to $300-500 \mathrm{fb}^{-1}$, while the barrel will perform up to $3000 \mathrm{fb}^{-1}$ with tolerable light loss. In the endcaps the response loss will impact the energy resolution and the energy equivalent electronics noise. The detection and trigger efficiencies will be seriously compromised over an increasingly large radius with respect to the beampipe. To maintain the best possible performance of the endcap region during HL-LHC, CMS plans to replace the endcaps before the start of the HL-LHC phase.

\section{Upgrade of the calorimeter endcaps}

The CMS hadron calorimeter endcap (HE) consists of brass absorber and plastic scintillator tiles. The scintillation light is collected by wavelength-shifting fibers. The tile transparency is affected by radiation damage such that a large fraction of the detector is expected to have a light output smaller than $5 \%$ of the initial value, by the end of the LHC operation $\left(300-500 \mathrm{fb}^{-1}\right)$. The plastic tiles must therefore be replaced before the start of the HL-LHC phase. The hadron calorimeter endcaps are located just behind the ECAL endcaps. Most of the services of the ECAL endcaps (cooling pipes, cables, optical fibres) run in front of the HE, so an intervention on the HE should preferably happen at the same time as the replacement of the ECAL endcaps. Two possible scenarios are considered: 
- if a replacement of the HE tiles is possible, while retaining the existing HE absorber, then the ECAL endcaps can be replaced by a new standalone detector, occupying the same volume.

- if both calorimeters must be replaced entirely, a combined design of the endcap calorimeters is possible, with the option of extending the coverage up to $|\eta|=4$.

The choice between the two scenarios will be driven by several factors including the performance, the cost, the time needed for dismounting and re-installation, and the activation of the original detectors.

One proposed design for the standalone endcap electromagnetic calorimeter is a sampling calorimeter made of lead or tungsten absorber plates and inorganic scintillator slices as active material. Recent developments in inorganic scintillators include crystals with improved resistance to hadron damage with respect to $\mathrm{PbWO}_{4}$, such as $\mathrm{LYSO}$ and $\mathrm{CeF}_{3}$ [9]. The scintillating light may be read-out in a shashlik configuration [10] with a wavelength shifting fiber crossing all crystal and absorber plates and carrying the light to a photo-diode at the rear. A research and development program is ongoing to develop sufficiently radiation-tolerant fibers and photo-detectors.

Two alternative solutions are considered for the combined calorimeter scen ario.

The first solution is based on the Dual Readout calorimetry [11] as proposed by the DREAM Collaboration. There are large fluctuations in the hadronic and electromagnetic components of a hadronic shower. Usually calorimeters do not compensate for this difference and give a very different response for the electromagnetic and hadronic components of a shower, thus spoiling the energy resolution. This effect can be corrected with the simultaneous read-out of both components in the same module. The proposed implementation is based on a sampling calorimeter made of tungsten or steel and two types of fibers as active material: scintillating fibers, and quartz fibers to detect Cerenkov light. A prototype module of the DREAM calorimeter was tested with pion and electron beams at CERN and the working principle of the dual read-out energy correction was demonstrated [11]. As for the case of the standalone shashlik calorimeter, the development of radiation hard fibers and photo-detectors is a key requirement for this solution. Detailed simulations are needed in order to study the performance of the dual readout calorimeter and optimize the fiber density for the HL-LHC application.

The second solution is an imaging calorimeter, as proposed for the Calice experiment at the Linear Collider [12]. The principle is to measure the charged particle momenta in the silicon tracker, and neutral particles in the calorimeter. The key point is to reconstruct even very closely spaced showers as separated objects through a finely granulated and longitudinally segmented calorimeter in order to match the calorimeter clusters to the silicon tracker tracks. The showers are reconstructed in the calorimeter through sophisticated pattern recognition programs that need to handle signals from the order of $10^{6}$ detector channels. The high rates in the ECAL endcap region, as high as $50 \mathrm{MHz} / \mathrm{cm}^{2}$ at high $\eta$, drive the choice of the detector type. Recent developments in low cost gaseous detectors, such as micro-megas and GEMs, may sustain these rates. Several considerations have to be addressed in order to employ this kind of detector at the HL-LHC. The imaging calorimeter approach needs to be tested with simulations of the nominal HL-LHC pile-up. The huge number of channels required for the imaging calorimeter demands inexpensive and compact electronics to be employed. The adoption of a digital or semi-digital read-out for the detector 
is proposed by the Calice Collaboration, where the signals are sampled with one or few bits respectively. However saturation and linearity of the detector are also concerns in this case. Cells must also be summed quickly to reconstruct shower energies for the Level-1 trigger. Another key point is the cooling, which is not a problem at the linear collider, where the detector is run in a pulsed mode, while at the LHC continuous running for several hours is expected.

\section{Pile-up mitigation techniques}

Pile-up is most critical in the forward region, so upgrades must be optimized for high pileup conditions. There will be an average of 130 concurrent events per bunch crossing. The event verteces will be distributed along the beam axis with a spread of about $5 \mathrm{~cm}$. This characteristic can be used to mitigate the pile-up using both the particle impact angle at the calorimeter and time of flight information.

The use of a dense, granular calorimeter will help to separate energy deposits, and longitudinal segmentation can be used to reconstruct the impact angle on the detector. High precision timing may help to identify or constrain the number of candidate verteces.

Pile-up mitigation through precision timing may improve particle reconstruction (removing the spurious energy deposits from reconstructed calorimeter clusters). It may permit particle to vertex matching prior to jet reconstruction, especially for neutral particles, and enable the emission vertex for the two-photon Higgs boson decay to be identified (improving the mass measurement resolution). The subdetector providing precision timing measurement should ideally be associated to the most granular detector: the electromagnetic calorimeter would certainly be the best choice in CMS. The desired resolution is $10-30$ ps. Some detectors have reached such resolution, namely the Micro-Channel-Plate photomultiplier tubes (MCP-PMT). Such a detector with time of flight measurement capability could be implemented as a preshower for the new endcap calorimeter or as one of the layers of the sampling calorimeter.

\section{Upgrade of the ECAL barrel}

In the barrel the neutron fluence will cause a growth in dark current for the APDs, which will increase the electronic noise proportionally to the square root of the current. A partial beneficial annealing of the current is expected during shutdowns. However, the noise increase per channel is expected to be in the range of $200-300 \mathrm{MeV}$, depending on $\eta$, after 3000/fb. A mitigation of the APD dark current of a factor about two could be obtained by lowering the operating temperature of the calorimeter from the present $18^{\circ} \mathrm{C}$ to $8-10^{\circ} \mathrm{C}$. The light output of $\mathrm{PbWO}_{4}$ crystals is slightly higher at lower temperature. However, the beneficial annealing of $\gamma$-induced crystal transparency loss has been observed to be slightly less effective at lower temperature. The time constant for the emission of scintillation light is also slower by about $10-20 \%$, which is still tolerable. The optimal operating temperature must therefore be chosen with respect to the overall performance. There are however technical considerations to be investigated relating to this option with respect primarily to the humidity and the dew point in the detector.

The first level (L1) of the current CMS trigger, composed of custom hardware processors, uses information from the calorimeters and muon detectors to select the most interesting events in a time 
interval of less than $4 \mu \mathrm{s}$. The High Level Trigger (HLT) processor farm further decreases the event rate from around $100 \mathrm{kHz}$ to around $300 \mathrm{~Hz}$, before data storage. In order to cope with the high pile-up it is foreseen to upgrade the L1 trigger for the HL-LHC, such that it will acquire information both from the tracker and the other sub-detectors. The processing of the tracker data will require $10 \mu \mathrm{s}$. However, the current ECAL front-end buffer has a maximum latency of $6.4 \mu$ s and cannot cope with this requirement. The ECAL front-end electronics must therefore be replaced. In the original ECAL electronics design, it was foreseen to read-out all channels at $40 \mathrm{MHz}$, transfer the data to the service cavern through optical links, and use fast processors to select events for the L1 trigger in the back-end. However, the optical links that were available at the time of construction were not fast enough and the number of required links was not affordable. The ECAL L1 trigger was therefore implemented by summing energy deposits from several channels on the detector front-end causing the L1 decision to be based on a coarse detector granularity.

Nowadays, the original design can be implemented with fewer optical links, with the advantage that the L1 trigger can base its decisions on information down to the individual channel level. This possibility, in conjunction with the information from the silicon tracker on the presence of a track matching the ECAL energy deposit, will improve substantially the rejection power against QCD events faking electron signals. Similarly, the absence of tracker hits may be exploited to select isolated photons. Complex trigger algorithms, with an electron in association with a jet, will also exploit the presence of the track trigger, with the possibility to check the consistency of the vertex of the electron and jet candidates and reduce fake jets from pile-up events.

In conclusion, the replacement of the ECAL front-end boards will bring substantial benefits to the CMS trigger system, allowing longer latency and better electron/photon trigger selection. However it must be stressed that the refurbishment of the ECAL electronics requires the dismounting of all the ECAL modules from the detector, which is a complex and long intervention.

\section{Conclusions}

The HL-LHC program will exploit the full potential of the LHC machine to precisely measure the couplings and rare decays of the Higgs boson, self-interaction through the double Higgs boson production, Vector Boson Scattering, and the properties of newly discovered particles.

The increase in luminosity poses severe requirements on the detectors in terms of performance and radiation tolerance.

The CMS electromagnetic calorimeter group has performed thorough tests of detector components to study the response evolution as a function of integrated luminosity. The crystal radiation damage, particularly due to hadron fluence, is an issue for the endcap calorimeter, which must be replaced after $500 \mathrm{fb}^{-1}$. New calorimeter options are being studied. Key points are radiation tolerance, granularity and segmentation. Precision timing may add important information for pile-up mitigation.

The ECAL barrel will perform up to $3000 \mathrm{fb}^{-1}$ with tolerable light loss, while the increase of the APD dark current will cause higher electronics noise. Lowering the barrel operating temperature may mitigate this effect. The replacement of the ECAL barrel front-end boards, allowing longer latency for the CMS trigger system, will also improve the electron/photon trigger selection. 


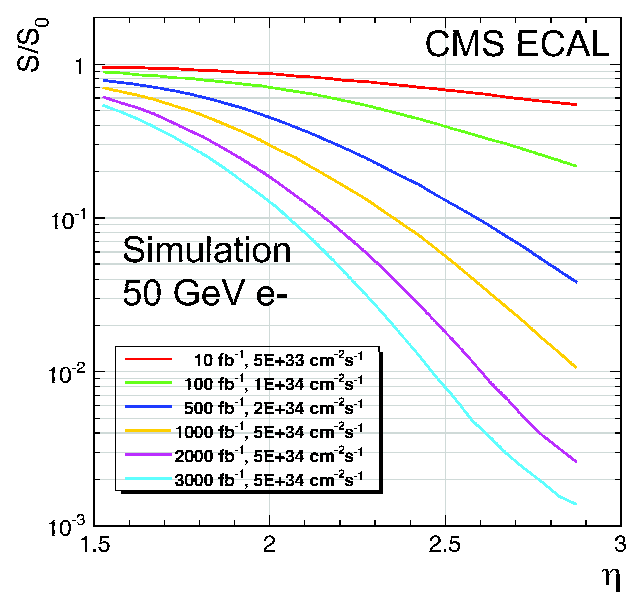

Figure 1: Light output variation for an electron shower of $50 \mathrm{GeV}$ with respect to the undamaged detector as a function of the pseudorapidity $\eta$ in the ECAL endcaps, and the detector ageing due to the radiation expected at the LHC.
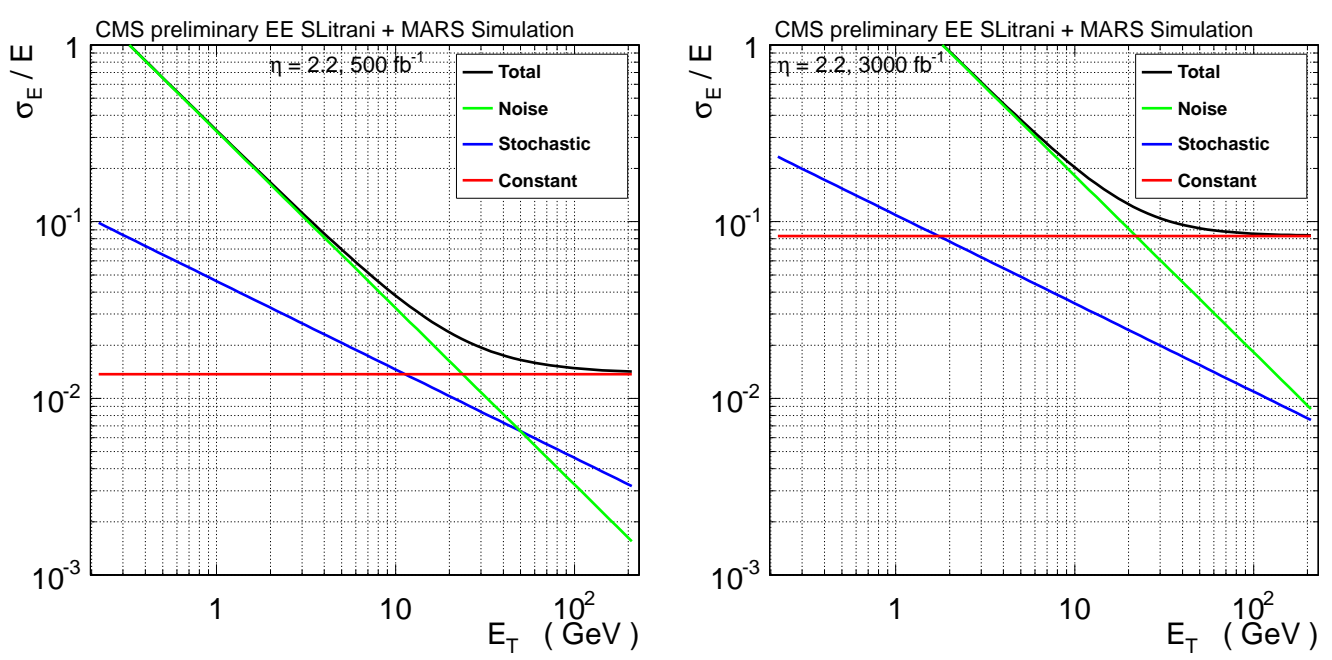

Figure 2: Energy resolution as a function of the transverse energy for unconverted photons impacting the calorimeter at pseudorapidity $|\eta|=2.2$, for a detector ageing corresponding to (left) $500 / \mathrm{fb}$ and (right) 3000/fb of integrated luminosity.

These planned upgrades are designed to provide the best possible triggering and object identification capabilities under HL-LHC conditions, in order to meet the HL-LHC physics goals.

\section{References}

[1] R. Adolphi et al, The CMS experiment at the CERN LHC, CMS Collaboration, JINST 0803:S08004 (2008).

[2] S. Chatrchyan et al. Observation of a new boson with mass near $125 \mathrm{GeV}$ in pp collisions at $\sqrt{s}=7$ and $8 \mathrm{TeV}$, CMS Collaboration, JHEP 1306 (2013) 081. 
[3] S. Chatrchyan et al., Energy calibration and resolution of the CMS electromagnetic calorimeter in pp collisions at $\sqrt{s}=7 \mathrm{TeV}$, JINST 8 P09009 (2013) [doi:10.1088/1748-0221/8/09/P09009].

[4] N. V. Mokhov, The MARS code system user guide, Fermilab-FN-628 (1995) and http://www-ap.fnal.gov/MARS

[5] S. Agostinelli, GEANT4 - A Simulation toolkit, Nucl. Instrum. Meth. A 506 (2003) 250. 10.1016/S0168-9002(03)01368-8.

[6] F.X. Gentit, LITRANI, CMS-TN-1996-143 (1996), and http://gentitfx.fr/SLitrani

[7] F. Nessi Tedaldi, Response evolution of the CMS ECAL and R\&D studies for electromagnetic calorimetry at the High-Luminosity LHC, 2012 IEEE Nuclear Science Symposium Conference, also available as CMS-CR-2012/296 [arxiv.org/pdf/1211.3885v1.pdf].

[8] D. E. Leslie, The effect of pulse rate on VPT response and the use of an LED light to improve stability, Proceedings of the 11th ICATPP Conference on Astroparticle, Particle, Space Physics, Detectors and Medical Applications, Como, (2009) CMS-CR-2009-284.

[9] G. Dissertori et al., NSS 2011 paper NP5.S-228.

[10] S. Amato et al., LHCb-TDR-2, LHCb calorimeters : Technical Design Report, CERN-LHCC-2000-0036.

[11] R. Wigmans, Dual-readout calorimetry - excellent precision for ALL particles, and NO calibration issues, these proceedings.

[12] M. Chefdeville, Development of highly granular calorimeters in CALICE, these proceedings. 